TRANSACTIONS OF THE

AMERICAN MATHEMATICAL SOCIETY

Volume 362, Number 7, July 2010, Pages 3691-3703

S 0002-9947(10)05116-0

Article electronically published on February 17, 2010

\title{
ALGEBRAIC CYCLES ON A PRODUCT OF TWO HILBERT MODULAR SURFACES
}

\author{
CRISTIAN VIRDOL
}

\begin{abstract}
In this paper we prove the Tate conjecture for a product of two Hilbert modular surfaces for non-CM submotives.
\end{abstract}

\section{INTRODUCTION}

Let $X$ be a smooth projective variety of dimension $n$ defined over a number field $F$ and let

$$
\bar{X}=X \times_{F} \overline{\mathbb{Q}} .
$$

For a prime number $l$, let $H_{e t}^{i}\left(X, \overline{\mathbb{Q}}_{l}\right)$ be the étale cohomology of $\bar{X}$. If $K$ is a number field, we denote $\Gamma_{K}:=\operatorname{Gal}(\overline{\mathbb{Q}} / K)$. The Galois group $\Gamma_{F}$ acts on $H_{e t}^{i}\left(X, \overline{\mathbb{Q}}_{l}\right)$ by a representation $\phi_{i, l}$. For any $j \in \mathbb{Z}$, let $H_{e t}^{i}\left(X, \overline{\mathbb{Q}}_{l}\right)(j)$ denote the representation of $\Gamma_{F}$ on $H_{e t}^{i}\left(X, \overline{\mathbb{Q}}_{l}\right)$ defined by $\phi_{i, l} \otimes \xi_{l}^{j}$, where $\xi_{l}$ is the $l$-adic cyclotomic character. The elements of $V^{i}(X, E):=\left(H_{e t}^{2 i}\left(X, \overline{\mathbb{Q}}_{l}\right)(i)\right)^{\Gamma_{E}}$ are called Tate cycles on $X$ defined over $E$. The union

$$
V^{i}(X):=\bigcup_{E} V^{i}(X, E)
$$

is the space of all Tate cycles on $X$.

To each algebraic subvariety $Y$ of $X$ of codimension $i$, one can associate a cohomology class

$$
[Y] \in H_{2 n-2 i}(X(\mathbb{C}), \mathbb{Q}) \cong H_{B}^{2 i}(X(\mathbb{C}), \mathbb{Q})(i),
$$

where $H_{B}^{2 i}(X(\mathbb{C}), \mathbb{Q})$ is the Betti cohomology. Then using the isomorphism

$$
H_{B}^{2 i}(X(\mathbb{C}), \mathbb{Q})(i) \otimes_{\mathbb{Q}} \mathbb{Q}_{l} \cong H_{e t}^{2 i}\left(X, \mathbb{Q}_{l}\right)(i),
$$

we obtain a class $[Y] \in H_{e t}^{2 i}\left(X, \mathbb{Q}_{l}\right)(i)$. A cohomology class $[Y]$ obtained in this way is called algebraic. If $Y$ is defined over an extension $E$ of $F$, then we obtain a class $[Y] \in H_{e t}^{2 i}\left(X, \mathbb{Q}_{l}\right)(i)^{\Gamma_{E}}$. The first part of the Tate conjecture states that every Tate cycle is algebraic.

The $L$-function $L^{2 i}\left(s, X_{/ F}\right)$ (more exactly, the Euler product) attached to the representation $\phi_{2 i, l}$ converges for $\operatorname{Re}(s)>i+1$. The second part of the Tate conjecture [TA] states that the $L$-function $L^{2 i}\left(s, X_{/ E}\right)$ has a meromorphic continuation to the entire complex plane and has a pole at $s=i+1$ of order equal to

$$
\operatorname{dim}_{\overline{\mathbb{Q}}_{l}} V^{i}(X, E)
$$

In their work [HLR, Harder, Langlands and Rapoport had proved the first part of the Tate conjecture for Hilbert modular surfaces for non-CM submotives. In

Received by the editors July 17, 2008.

2000 Mathematics Subject Classification. Primary 11R42, 11R80.

(C)2010 American Mathematical Society Reverts to public domain 28 years from publication 
[K] and $\mathrm{MR}$ ] the first part of the Tate conjecture was proved for Hilbert modular surfaces for CM submotives, and thus using the two results, one gets the full first part of the Tate conjecture asserting the algebraicity of all the Tate cycles of Hilbert modular surfaces over an arbitrary number field. The second part of the Tate conjecture for Hilbert modular surfaces was also proved in $\underline{\mathrm{HLR}}, \mathrm{K}$ ] and $\mathrm{MR}$, but only for solvable number fields.

In this paper we consider a quadratic real field $F$ and let $G=\operatorname{Res}_{F / \mathbb{Q}} G L_{2 / F}$. Let $S_{K_{i}}:=S_{G, K_{i}}$ be the Hilbert modular surface associated to an open compact subgroup $K_{i}$ of $G\left(\mathbb{A}_{f}\right)$, for $i=1,2$, where $\mathbb{A}_{f}$ is the finite part of the ring of adeles $\mathbb{A}_{\mathbb{Q}}$ of $\mathbb{Q}$. In this paper we prove the first part of the Tate conjecture for $S_{K_{1}} \times S_{K_{2}}$ for the tensor product of non-CM submotives of the individual factors $S_{K_{1}}$ and $S_{K_{2}}$ (see Theorem 7.1 for details). We also prove the second part of the Tate conjecture for $S_{K_{1}} \times S_{K_{2}}$, but only for solvable number fields (see Proposition 8.1).

We remark that in MP, the space of Tate cycles on the product of two arbitrary Hilbert modular surfaces was computed in terms of automorphic representations including the exact determination of their fields of definition, but in $\mathrm{MP}$ the algebraicity of all Tate cycles was not proved, but only of those spanned by the tensor product of algebraic cycles on individual factors, which from [HLR, [K] and MR we know to be algebraic.

We remark that the first part of the Tate conjecture is also known for the non$\mathrm{CM}$ submotives of the Shimura surfaces treated in [LA and [FH], corresponding to a quadratic real field $F$ and to a quaternion algebra $D=B \otimes_{\mathbb{Q}} F$, where $B$ is a quaternion algebra over $\mathbb{Q}$, such that $D$ splits at the real places. Let $H$ be the algebraic group over $F$ associated to $D^{\times}$and let $G^{\prime}=\operatorname{Res}_{F / \mathbb{Q}} H$. Let $\mathbf{S}_{K_{i}}:=S_{G^{\prime}, K_{i}}$ be the quaternionic Shimura surface corresponding to an open compact subgroup $K_{i}$ of $G^{\prime}\left(\mathbb{A}_{f}\right)$, for $i=1,2$. Then the surfaces $\mathbf{S}_{K_{i}}$ for $i=1,2$ are compact. In the same way as we do in this paper, one can prove the first part of the Tate conjecture for $\mathbf{S}_{K_{1}} \times \mathbf{S}_{K_{2}}$ for the tensor product of non-CM submotives of the individual factors $\mathbf{S}_{K_{1}}$ and $\mathbf{S}_{K_{2}}$. We remark that because the quaternionic Shimura surfaces are compact, one has to replace the intersection cohomology in this paper by the étale cohomology and everything else remains unchanged. The second part of the Tate conjecture for $\mathbf{S}_{K_{1}} \times \mathbf{S}_{K_{2}}$ is also true for solvable number fields.

\section{Hilbert modular SURFaces}

Let $F$ be a real quadratic field and let $G=\operatorname{Res}_{F / \mathbb{Q}} G L_{2 / F}$. For $K$ a sufficiently small open compact subgroup of $G\left(\mathbb{A}_{f}\right)$, let $S_{K}$ be the smooth toroidal compactification of an open surface $S_{K}^{0}$ which satisfies

$$
S_{K}^{0}(\mathbb{C})=G(\mathbb{Q}) \backslash G\left(\mathbb{A}_{\mathbb{Q}}\right) / K_{\infty} K,
$$

where $K_{\infty}=S O_{2}(\mathbb{R}) \mathbb{R}^{\times} \times S O_{2}(\mathbb{R}) \mathbb{R}^{\times} \subset G(\mathbb{R})$. Then $S_{K}$ is a surface defined over $\mathbb{Q}$ and it is called a Hilbert modular surface. Let $\mathbf{S}=S_{1} \times S_{2}$, where $S_{i}=S_{K_{i}}$ is the Hilbert modular surface corresponding to a sufficiently small open compact subgroup $K_{i}$ of $G\left(\mathbb{A}_{f}\right)$, for $i=1,2$.

\section{Cohomology for Hilbert modular surfaces}

If $\pi$ is a cuspidal automorphic representation of weight 2 of $G L(2) / F$, then from Taylor [T], we know that there exists a $\lambda$-adic representation (for $\lambda$ a prime of the 
field of coefficients $\mathbf{O}$ of $\pi$, such that $\lambda \mid l$ for some rational prime $l$ )

$$
\rho_{\pi, \lambda}: \Gamma_{F} \rightarrow G L_{2}\left(\mathbf{O}_{\lambda}\right) \hookrightarrow G L_{2}\left(\overline{\mathbb{Q}}_{l}\right),
$$

which satisfies $L_{v}(s, \pi)=L_{v}\left(s, \rho_{\pi, \lambda}\right)$ for almost all finite places $v$ of $F$ and is unramified outside the primes dividing $\mathbf{n} l$, where $\mathbf{n}$ is the level of $\pi$. Here if we fix an isomorphism $i: \overline{\mathbb{Q}}_{l} \rightarrow \mathbb{C}$ and if $\rho_{\pi, \lambda}$ is unramified at $v$, then

$$
L_{v}\left(s, \rho_{\pi, \lambda}\right)=\operatorname{det}\left(1-i\left(\rho_{\pi, \lambda}\left(\operatorname{Frob}_{v}\right)\right) N v^{-s}\right)^{-1},
$$

where $\mathrm{Frob}_{v}$ is a geometric Frobenius. In order to simplify the notation we denote by $\rho_{\pi}$ the representation $\rho_{\pi, \lambda}$.

Let $K$ be a sufficiently small open compact subgroup of $G\left(\mathbb{A}_{f}\right)$. Then we have a decomposition

$$
H_{e t}^{2}\left(S_{K}, \overline{\mathbb{Q}}_{l}\right)=I H_{e t}^{2}\left(S_{K}, \overline{\mathbb{Q}}_{l}\right) \oplus H^{2}\left(S_{K}^{\infty}, \overline{\mathbb{Q}}_{l}\right),
$$

where $I H_{e t}^{2}\left(S_{K}, \overline{\mathbb{Q}}_{l}\right)$ is the intersection cohomology of the Baily-Borel compactification $\bar{S}_{K}$ of $S_{K}^{0}$, and $S_{K}^{\infty}$ is the divisor at infinity (a finite set of cusps) such that $\bar{S}_{K}=S_{K}^{0} \cup S_{K}^{\infty}$, and is defined by

$$
I H_{e t}^{2}\left(S_{K}, \mathbb{Q}_{l}\right):=\operatorname{Im}\left(H_{e t}^{2}\left(\bar{S}_{K}, \mathbb{Q}_{l}\right) \rightarrow H_{e t}^{2}\left(S_{K}^{0}, \mathbb{Q}_{l}\right)\right) .
$$

If $l$ is a prime number, let $\mathbb{H}_{K}$ be the Hecke algebra generated by the bi- $K$ invariant $\overline{\mathbb{Q}}_{l}$-valued compactly supported functions on $G\left(\mathbb{A}_{f}\right)$ under the convolution. If $\pi=\pi_{f} \otimes \pi_{\infty}$ is an automorphic representation of $G\left(\mathbb{A}_{\mathbb{Q}}\right)$, we denote by $\pi_{f}^{K}$ the space of $K$-invariants in $\pi_{f}$. The Hecke algebra $\mathbb{H}_{K}$ acts on $\pi_{f}^{K}$.

We have an action of the Hecke algebra $\mathbb{H}_{K}$ and an action of the Galois group $\Gamma_{\mathbb{Q}}$ on the intersection cohomology $I H_{e t}^{2}\left(S_{K}, \overline{\mathbb{Q}}_{l}\right)$ and these two actions commute. Then we know (see, for example, proposition 1.8 of [RT]):

Proposition 3.1. The representation of $\Gamma_{\mathbb{Q}} \times \mathbb{H}_{K}$ on the intersection cohomology $I H_{\text {et }}^{2}\left(S_{K}, \overline{\mathbb{Q}}_{l}\right)(1)$ is isomorphic to

$$
\bigoplus_{\pi} \rho(\pi) \otimes \pi_{f}^{K}
$$

where $\rho(\pi)$ is a representation of the Galois group $\Gamma_{\mathbb{Q}}$. The above sum is over weight 2 cuspidal automorphic representations $\pi$ of $G\left(\mathbb{A}_{\mathbb{Q}}\right)$, such that $\pi_{f}^{K} \neq 0$ and the $\mathbb{H}_{K}$-representations $\pi_{f}^{K}$ are irreducible and mutually inequivalent.

If $K^{\prime}$ and $K$ are open compact subgroups of $G\left(\mathbb{A}_{f}\right)$ such that $K^{\prime} \subseteq K$, then we obtain a finite morphism $S_{K^{\prime}} \rightarrow S_{K}$, and if $g \in G\left(\mathbb{A}_{f}\right)$, we obtain a morphism $S_{K} \rightarrow S_{g K g^{-1}}$. We consider the inverse limit

$$
S(\mathbb{C}):=\lim _{\overleftarrow{K}} S_{K}(\mathbb{C})=G(\mathbb{Q}) \backslash G\left(\mathbb{A}_{\mathbb{Q}}\right) / K_{\infty}
$$

The scheme $S$ has a $G\left(\mathbb{A}_{f}\right)$-action that satisfies:

$$
S / K=S_{K}
$$

In this paper it is convenient to consider the direct limit of $I H_{e t}^{2}\left(S_{K}, \overline{\mathbb{Q}}_{l}\right)$ as $K$ shrinks to the identity. We have

$$
I H_{e t}^{2}\left(S, \overline{\mathbb{Q}}_{l}\right)=\lim _{\vec{K}} I H_{e t}^{2}\left(S_{K}, \overline{\mathbb{Q}}_{l}\right)
$$

and

$$
I H_{e t}^{2}\left(S, \overline{\mathbb{Q}}_{l}\right)^{K}=I H_{e t}^{2}\left(S_{K}, \overline{\mathbb{Q}}_{l}\right) .
$$


Then, from Proposition 3.1, we get

$$
I H_{e t}^{2}\left(\bar{S}, \overline{\mathbb{Q}}_{l}\right)(1)=\bigoplus_{\pi} \rho(\pi) \otimes \pi_{f} .
$$

Since the representation $\rho(\pi)$ is semisimple (see $\S 4$ of [HLR] or corollary 3.8 of G] ) and from [MP, for example, we know that $\rho(\pi)$ is a subrepresentation of

$$
\operatorname{Ind}_{\Gamma_{F}}^{\Gamma_{\mathbb{Q}}}\left(\rho_{\pi} \otimes \rho_{\pi}^{\tau}\right)
$$

that satisfies

$$
\left.\rho(\pi)\right|_{\Gamma_{F}}=\rho_{\pi} \otimes \rho_{\pi}^{\tau},
$$

where $\tau$ is the nontrivial automorphism of $F$ over $\mathbb{Q}$, and $\rho_{\pi}^{\tau}$ is defined by

$$
\rho_{\pi}^{\tau}(\gamma)=\rho_{\pi}\left(\tau \gamma \tau^{-1}\right) .
$$

3.1. Betti cohomology. We define the cuspidal part of the Betti cohomology:

$$
H_{B, \text { cusp }}^{2}\left(S_{K}\right):=\operatorname{Im}\left(H^{2}\left(\bar{S}_{K}(\mathbb{C}), \mathbb{Q}\right) \rightarrow H^{2}\left(S_{K}^{0}(\mathbb{C}), \mathbb{Q}\right)\right),
$$

where $\bar{S}_{K}$ is the Baily-Borel compactification of $S_{K}^{0}$.

In this paper it is convenient to consider the direct limit of $H_{B, \text { cusp }}^{2}\left(S_{K}\right)$ as $K$ shrinks to the identity. We have

$$
H_{B, \text { cusp }}^{2}(S)=\lim _{\vec{K}} H_{B, \text { cusp }}^{2}\left(S_{K}\right) .
$$

Then

$$
H_{B, \text { cusp }}^{2}(S)^{K}=H_{B, \text { cusp }}^{2}\left(S_{K}\right) .
$$

There exists the Hodge decomposition:

$$
H_{B, \text { cusp }}^{2}\left(S_{K}\right) \otimes_{\mathbb{Q}} \mathbb{C}=\bigoplus_{p+q=2} H^{p, q}\left(S_{K}\right),
$$

where $H^{p, q}\left(S_{K}\right)=H^{p}\left(S_{K}(\mathbb{C}), \Omega^{q}\right)$, with $\Omega^{q}$ the sheaf of holomorphic $q$-forms.

We have the canonical isomorphisms:

$$
\Phi_{e t, K}: H_{B, \text { cusp }}^{2}\left(S_{K}\right) \otimes_{\mathbb{Q}} \mathbb{Q}_{l} \rightarrow I H_{e t}^{2}\left(S_{K}, \mathbb{Q}_{l}\right)
$$

and

$$
\Phi_{e t}: H_{B, \text { cusp }}^{2}(S) \otimes_{\mathbb{Q}} \mathbb{Q}_{l} \rightarrow I H_{e t}^{2}\left(S, \mathbb{Q}_{l}\right) .
$$

We denote by $V_{l}(\pi)$ the $\pi$-component of $I H_{e t}^{2}\left(S, \mathbb{Q}_{l}\right)(1)$ in the decomposition of Proposition 3.1 and by $V_{B}(\pi)$ the corresponding $\pi$-component of $H_{B \text {,cusp }}^{2}(S)$. Thus

$$
V_{B}(\pi) \otimes_{\mathbb{Q}} \mathbb{Q}_{l} \cong V_{l}(\pi) .
$$

For $s=\left(s_{1}, s_{2}\right)$, with each $s_{1}$ and $s_{2}$ being + or - , we can define a real analytic automorphism $\tau_{s}$ of $F \otimes \mathbb{C}-F \otimes \mathbb{R} \cong \mathbb{C}^{2}-\mathbb{R}^{2}$ by

$$
\tau_{s}\left(z_{1}, z_{2}\right)=\left(\tau_{s_{1}}\left(z_{1}\right), \tau_{s_{2}}\left(z_{2}\right)\right),
$$

where $\tau_{s_{j}}$ is the identity, respectively complex conjugation, if $s_{j}$ is + , respectively -. Each such involution $\tau_{s}$ acts on the Hilbert modular surface $S$ and its Betti cohomology. It also commutes with the Hecke action of $G\left(\mathbb{A}_{f}\right)$ and we obtain a decomposition

$$
V_{B}(\pi) \cong \bigoplus_{s \in \Sigma} V_{B}(\pi)^{s},
$$


where $\Sigma=\{s=( \pm, \pm)\}$ and $V_{B}(\pi)^{s}$ denotes the $s$-eigenspace of $V_{B}(\pi)$. Obviously $\Sigma$ forms a group under componentwise multiplication with identity $(+,+)$ and each eigenspace $V_{B}(\pi)^{s}$ is one-dimensional.

\section{TWISTING CORRESPONDENCE}

In this section we define a certain twisting correspondence on Hilbert modular surfaces (see $\S 2$ of $[\mathrm{MR}]$ for details). Let $\pi$ be a cuspidal automorphic representation of weight 2 with conductor $\mathfrak{a}=\mathfrak{a}(\pi)$, which is an integral ideal in $F$. Write $\mathfrak{a}_{v}=$ $\mathfrak{u}_{v}^{n_{v}(\mathfrak{a})} \mathfrak{o}_{v}, n_{v}(\mathfrak{a})=\operatorname{ord}_{v}(\mathfrak{a}) \geq 0$, and set

$$
K(\pi)=K_{1}(\mathfrak{a})=\prod_{v} K_{1}\left(\mathfrak{a}_{v}\right),
$$

where

$$
K_{1}\left(\mathfrak{a}_{v}\right)=\left\{\left(\begin{array}{ll}
a & b \\
c & d
\end{array}\right) \in G L_{2}\left(\mathfrak{a}_{v}\right) \mid c,(d-1) \in \mathfrak{u}_{v}^{n_{v}(\mathfrak{a})} \mathfrak{o}_{v}\right\}
$$

(when $n_{v}(\mathfrak{a})$ is zero, $K_{1}\left(\mathfrak{a}_{v}\right)$ is equal to $G L\left(2, \mathfrak{o}_{v}\right)$ ).

One knows that $\mathfrak{a}$ is the smallest of all the non-zero integral ideals $\mathfrak{i}$ in $O_{F}$ such that $\operatorname{dim} \pi_{f}^{K_{1}(\mathfrak{i})} \neq 0$. Furthermore we have $\operatorname{dim} \pi_{f}^{K_{1}(\mathfrak{i})}=1$.

Let $\mu$ be any finite order character of the group $I_{F}$ of ideles of $F$ of conductor $\mathfrak{c}$. Let $K=K_{1}(\mathfrak{a})$. Set

$$
K[\mu]:=K_{1}\left(\operatorname{lcm}\left(\mathfrak{a}, \mathfrak{c}^{2}\right)\right) .
$$

Let $\mathfrak{D}_{c}$ be the ring of integers of $F_{\mathfrak{c}}:=\prod_{v \mid \mathfrak{c}} F_{v}$, and let $X$ be the subset of $F_{\mathfrak{c}}$ defined by

$$
X:=\left\{x=\left(x_{v}\right) \in F_{\mathfrak{c}} \mid v\left(x_{v}\right) \geq-v(\mathfrak{c}), \forall v\right\} .
$$

Let $\tilde{X}$ be a set of representatives in $X$ for $X \bmod \mathfrak{D}_{\mathfrak{c}}$, which is a group isomorphic to $\mathfrak{D}_{\mathfrak{c}} / \mathfrak{c} \mathfrak{D}_{\mathfrak{c}}$. For each $t$ in $\tilde{X}$, we define $u(t)=\left(\begin{array}{ll}1 & t \\ 0 & 1\end{array}\right)$. We have that

$$
S_{K}^{0}(\mathbb{C})=\bigcup_{j=1}^{h(K)} \Gamma_{j} \backslash(\mathbb{C}-\mathbb{R})^{2},
$$

with

$$
\Gamma_{j}=G(\mathbb{Q}) \cap x_{j} G(\mathbb{R})^{+} K x_{j}^{-1},
$$

where

$$
x_{j}=\left(\begin{array}{cc}
b_{j} & 0 \\
0 & 1
\end{array}\right),
$$

where $\mathbb{A}_{F}^{\times}=\bigcup_{j=1}^{h(K)} F^{\times} b_{j} F_{\infty}^{+} \operatorname{det} K$, with $b_{j} \in \mathbb{A}_{F, f}$, and $F_{\infty}^{\times}$is the subset of $F \otimes \mathbb{R} \cong$ $\mathbb{R}^{2}$ consisting of totally positive elements, and $G(\mathbb{R})^{+}$is the subgroup of $G(\mathbb{R})$ consisting of totally positive elements.

For every $x$ in $G\left(\mathbb{A}_{f}\right)$ we have the usual Hecke correspondence $T(x)$ on $S_{K}^{0}$, which depends only on the double coset $K g K$, and is given by $T(x)(g)=$ $R(x)\left(R(1)^{-1}(g)\right)$, where if $K_{x}:=K \cap x K x^{-1}$, then $R(1): S_{K_{x}}^{0} \rightarrow S_{K}^{0}$ and $R(x): S_{K_{x}}^{0} \rightarrow S_{K}^{0}$, are obtained from the two homomorphisms $K_{x} \rightarrow K$ given by the inclusion and by conjugation by $x^{-1}$. Then $T(x)$ does not in general preserve the connected components $S_{K}^{(j)}{ }^{0}$ of $S_{K}^{0}$. Let $T_{j}(x)$ denote the restriction of $T(x)$ to $S_{K}^{(j)^{0}}$ when $\operatorname{det}(x)=1$. 
We define the twisting correspondence $R(\mu) \subset S_{K}^{0} \times S_{K[\mu]}^{0}$ by

$$
R(\mu)=\sum_{j=1}^{h(K)} \mu_{f}\left(\operatorname{det}\left(x_{j}\right)\right) R_{j}(\mu),
$$

with

$$
R_{j}(\mu)=\sum_{t \in \tilde{X}} T_{j}\left(u_{t}\right)
$$

Then for all $x \in G\left(\mathbb{A}_{f}\right)$, we have

$$
T(x) \circ R(\mu)=\mu_{f}(\operatorname{det}(x)) R(\mu) \circ T(x) .
$$

We remark that the correspondences $R_{j}(\mu)$ and $R(\mu)$ could be extended to the

Baily-Borel compactifications $S_{K}^{j}$ and $S_{K}$ (obtained by adding cusps to $S_{K}^{(j)^{0}}$ and $S_{K}^{0}$ ). The twisting correspondence is algebraic and acts on any cohomology group, Betti or étale, of the surface $S=\lim S_{K}$ and of the variety $S \times S$, and is compatible under the isomorphisms $\Phi_{e t, K}$ and $\Phi_{e t}$, and $\Phi_{e t, K} \times \Phi_{e t, K[\mu]}$ and $\Phi_{e t} \times \Phi_{e t}$ defined in $\S 3$. The induced operator sends the $\pi$-component to the $\pi \otimes \mu$-component. The twisting correspondence $R(\mu)$ is rational over the abelian extension $\mathbb{Q}^{\left.\mu\right|_{I_{\mathbb{Q}}}}$ of $\mathbb{Q}$, determined by $\left.\mu\right|_{I_{\mathbb{Q}}}$, where $I_{\mathbb{Q}}$ is the ideles group of $\mathbb{Q}$. We denote by $Z_{g_{1}, g_{2}}(\mu)$ the algebraic cycle of codimension 2 of $S_{K} \times S_{K[\mu]}$ induced by $T\left(g_{2}\right) \circ R(\mu) \circ T\left(g_{1}\right)$, where $g_{1}, g_{2} \in G\left(\mathbb{A}_{f}\right)$.

If $\mu$ is a finite order character of $F$, then its component at any Archimedean place is 1 or the sign character, and we define the signature $s(\mu)$ of $\mu$ to be $\left(s(\mu)_{1}, s(\mu)_{2}\right)$, where $s(\mu)_{j}$ is the sign of $\mu_{1}$, resp. $\mu_{\tau}$ (here $\tau$ is the non-trivial automorphism of $F$ over $\mathbb{Q}$ ) for $j=1$ or 2 (at any Archimedean place $u$, the sign of $\mu_{u}$ is + , resp. - , if $\mu_{u}$ is trivial, resp. non-trivial).

It is easy to see that:

Lemma 4.1. The twisting correspondence $R(\mu)$ sends a vector in $V_{B}(\pi)^{s}$ into $V_{B}(\pi \otimes \mu)^{s s(\mu)}$.

\section{KNOWN RESULTS}

It is known that (see for example proposition 4.5 .4 of [HLR] :

Proposition 5.1. If $\pi$ is a cuspidal automorphic representation of weight 2 of $G L(2) / F$, where $F$ is a totally real field, then one of the following two statements holds:

(i) $\left.\rho_{\pi}\right|_{\Gamma_{L}}$ is irreducible for each finite extension $L / F$.

(ii) There exists a quadratic extension $L / F$ and an algebraic Hecke character $\psi$ of $L$ such that $\rho_{\pi} \cong \operatorname{Ind}_{L}^{F}(\psi)$.

We say that a representation $\rho$ of a group $G$ is dihedral if there exists a normal subgroup $N$ of index 2 in $G$ and a character $\psi: N \rightarrow \mathbb{C}^{\times}$such that $\rho=\operatorname{Ind}_{N}^{G} \psi$.

We say that an automorphic representation $\pi$ of $G L(2) / L$ for some number field $L$ is of CM type if there exists some quadratic Galois character $\eta: I_{L} / L^{\times} \rightarrow \mathbb{C}^{\times}$, where $I_{L}$ is the ideles group of $L$, with $\eta \neq 1$ such that $\pi \cong \pi \otimes \eta$. If $\pi$ is an automorphic representation of weight 2 of $G L(2) / L$, then $\pi$ is of CM type if and only if $\rho_{\pi}$ is a dihedral representation. 
We know the following result (theorem 2.1 of $[\mathrm{MP}]$ ):

Proposition 5.2. The tensor product of two 2-dimensional irreducible complex representations of a group is reducible only if either both representations are dihedral or they are the twist of each other by a character.

We know (lemma 4.2 of $[\mathrm{MP}]$ ):

Proposition 5.3. Let $\pi_{1}$ and $\pi_{2}$ be two cuspidal non-CM representations of $G L(2) /$ $F$, where $F$ is a totally real field. Suppose that $\pi_{1}$ and $\pi_{2}$ are twists of each other over an extension of $F$. Then $\pi_{1}$ and $\pi_{2}$ are twists of each other over $F$.

We know (Proposition 4.1 of [MP]):

Proposition 5.4. Suppose that $\pi$ is a cuspidal, non-CM automorphic representation of $G L(2) / K$ for some finite extension $K / \mathbb{Q}$. Suppose that $K$ is a quadratic extension of $k$ and $\tau$ is the automorphism of $K$ over $k$. If $\pi^{\tau} \cong \pi \otimes \chi$ for a Hecke character $\chi$ of $K$, then $\chi$ is trivial when restricted to the ideles of $k$.

We know (Corollary 2.6 of $\underline{\mathrm{MP}}$ ):

Proposition 5.5. Let $\rho$ be a 2-dimensional irreducible representation of a group $G$. Then $\operatorname{Sym}^{2}(\rho)$ is reducible if and only if $\rho$ is dihedral.

We know (see the main theorem of [GJ]):

Proposition 5.6. Let $\pi$ be a cuspidal, non-CM automorphic representation of weight 2 of $G L(2) / K$ for some finite extension $K / \mathbb{Q}$. Then $S y m^{2} \pi$ is a cuspidal automorphic representation of $G L(3) / K$.

We know (lemma 2.9 of $[\mathrm{MP}]$ ):

Proposition 5.7. For 2-dimensional irreducible non-dihedral representations $\sigma_{1}$ and $\sigma_{2}$ of a group $G$, Sym $^{2} \sigma_{1} \cong S y m^{2} \sigma_{2}$ if and only if $\sigma_{1} \cong \sigma_{2} \otimes \chi$ for a quadratic character $\chi$ of $G$.

We know ([JPSS]):

Proposition 5.8. If $\pi_{1}$ and $\pi_{2}$ are two cuspidal unitary automorphic representations of $G L(n) / L$ and $G L(m) / L$, where $L$ is a number field, then the function $L\left(s, \pi_{1} \times \pi_{2}\right)$ satisfies a functional equation and is meromorphic with possible poles only at $s=0$ and 1 , and does not vanish at $s=1$. The function $L\left(s, \pi_{1} \times \pi_{2}\right)$ is holomorphic iff $\pi_{1} \nsucceq \pi_{2}^{*}$, and if $\pi_{1} \cong \pi_{2}^{*}$, then it has a pole of order 1 at $s=1$.

\section{TATE CYCLES}

Let $S_{1}:=S_{K_{1}}$ be the Hilbert modular surface associated to some sufficiently small open compact subgroup $K_{1}$ of $G\left(\mathbb{A}_{f}\right)$ and $S_{2}:=S_{K_{2}}$ be the Hilbert modular surface associated to some sufficiently small open compact subgroup $K_{2}$ of $G\left(\mathbb{A}_{f}\right)$. By the Künneth formula we have

$$
I H_{e t}^{4}\left(S_{1} \times S_{2}, \overline{\mathbb{Q}}_{l}\right)(2)=\bigoplus_{i+j=4} I H_{e t}^{i}\left(S_{1}, \overline{\mathbb{Q}}_{l}\right)(1) \otimes I H_{e t}^{j}\left(S_{2}, \overline{\mathbb{Q}}_{l}\right)(1) .
$$

Also we have a decomposition:

$$
H_{B, \text { cusp }}^{4}\left(S_{1} \times S_{2}\right)=\bigoplus_{i+j=4} H_{B, \text { cusp }}^{i}\left(S_{1}\right) \otimes H_{B, \text { cusp }}^{j}\left(S_{2}\right) .
$$


We have the canonical isomorphism:

$$
\Phi_{e t}: H_{B, \text { cusp }}^{4}\left(S_{1} \times S_{2}\right) \otimes_{\mathbb{Q}} \mathbb{Q}_{l} \rightarrow I H_{e t}^{4}\left(S_{1} \times S_{2}, \mathbb{Q}_{l}\right),
$$

which is compatible with the above decompositions.

The essential part of the above intersection cohomology decomposition is

$$
I H_{e t}^{2}\left(S_{1}, \overline{\mathbb{Q}}_{l}\right)(1) \otimes I H_{e t}^{2}\left(S_{2}, \overline{\mathbb{Q}}_{l}\right)(1) .
$$

From Proposition 3.1, we obtain

$$
\begin{aligned}
& I H_{e t}^{2}\left(S_{1}, \overline{\mathbb{Q}}_{l}\right)(1) \otimes I H_{e t}^{2}\left(S_{2}, \overline{\mathbb{Q}}_{l}\right)(1) \\
= & \left(\bigoplus_{\pi_{1}} V\left(\pi_{1}\right) \otimes \pi_{1 f}^{K_{1}}\right) \otimes\left(\bigoplus_{\pi_{2}} V\left(\pi_{2}\right) \otimes \pi_{2 f}^{K_{2}}\right) \\
= & \bigoplus_{\pi_{1}, \pi_{2}}\left(V\left(\pi_{1}\right) \otimes V\left(\pi_{2}\right)\right) \otimes\left(\pi_{1 f}^{K_{1}} \otimes \pi_{2 f}^{K_{2}}\right),
\end{aligned}
$$

where $\pi_{1}$ and $\pi_{2}$ run over the set of cuspidal automorphic representations of $G\left(\mathbb{A}_{\mathbb{Q}}\right)$. The group $\Gamma_{\mathbb{Q}}$ acts on each summand above by $\rho\left(\pi_{1}\right) \otimes \rho\left(\pi_{2}\right) \otimes 1$.

For an extension $k$ of $\mathbb{Q}$ we must compute the $\Gamma_{k}$-invariant subspace of $V\left(\pi_{1}\right) \otimes$ $V\left(\pi_{2}\right)$, which is isomorphic to

$$
\operatorname{Hom}_{\overline{\mathbb{Q}}_{l}\left[\Gamma_{k}\right]}\left(V\left(\pi_{1}\right), V\left(\pi_{2}\right)^{*}\right),
$$

which is isomorphic (see the computation of $\rho\left(\pi_{2}\right)$ from $\S 3$ ) to

$$
\operatorname{Hom}_{\overline{\mathbb{Q}}_{l}\left[\Gamma_{k}\right]}\left(V\left(\pi_{1}\right), V\left(\pi_{2}^{*}\right)\right) \text {. }
$$

For $k$ a finite extension of $\mathbb{Q}$, define:

$$
\mathbf{V}\left(\pi_{1}, \pi_{2}, k\right):=\left\{x \in V\left(\pi_{1}\right) \otimes V\left(\pi_{2}\right) \mid \rho\left(\pi_{1}\right) \otimes \rho\left(\pi_{2}\right)(a) x=x \text {, for all } a \in \Gamma_{k}\right\} .
$$

The elements of $\mathbf{V}\left(\pi_{1}, \pi_{2}, k\right)$ are called Tate cycles defined over $k$. We denote by $\mathbf{U}\left(\pi_{1}, \pi_{2}, k\right) \subseteq \mathbf{V}\left(\pi_{1}, \pi_{2}, k\right)$ the subspace of algebraic cycles defined over $k$.

For $\nu$ a finite order character of $\Gamma_{\mathbb{Q}}$, define:

$$
\mathbf{V}\left(\pi_{1}, \pi_{2} ; \nu\right):=\left\{x \in V\left(\pi_{1}\right) \otimes V\left(\pi_{2}\right) \mid \rho\left(\pi_{1}\right) \otimes \rho\left(\pi_{2}\right)(a) x=\nu^{-1}(a) x, \text { for all } a \in \Gamma_{\mathbb{Q}}\right\}
$$

and

$$
\mathbf{V}\left(\pi_{1}, \pi_{2}, \mathbb{Q}^{a b}\right):=\bigcup_{\nu} \mathbf{V}\left(\pi_{1}, \pi_{2} ; \nu\right) .
$$

Let $\mathbf{U}\left(\pi_{1}, \pi_{2} ; \nu\right) \subseteq \mathbf{V}\left(\pi_{1}, \pi_{2} ; \nu\right)$ and $\mathbf{U}\left(\pi_{1}, \pi_{2}, \mathbb{Q}^{a b}\right) \subseteq \mathbf{V}\left(\pi_{1}, \pi_{2}, \mathbb{Q}^{a b}\right)$ be the subspaces of algebraic cycles. We remark that in the cases treated in this paper, i.e., when either $\pi_{1}$ or $\pi_{2}$ is non-CM, for $k$ sufficiently large we have $\mathbf{V}\left(\pi_{1}, \pi_{2}, k\right)=$ $\mathbf{V}\left(\pi_{1}, \pi_{2} ; \nu\right)$ for some finite order character $\nu$ of $\Gamma_{\mathbb{Q}}$; i.e., for $k$ sufficiently large we have $\mathbf{V}\left(\pi_{1}, \pi_{2}, k\right)=\mathbf{V}\left(\pi_{1}, \pi_{2}, \mathbb{Q}^{a b}\right)$, i.e. all the Tate cycles are defined over abelian extensions of $\mathbb{Q}$. When both $\pi_{1}$ and $\pi_{2}$ are CM, it is possible to have for all $k$ that $\mathbf{V}\left(\pi_{1}, \pi_{2}, k\right) \neq \mathbf{V}\left(\pi_{1}, \pi_{2}, \mathbb{Q}^{a b}\right)$.

\section{TAte COnjeCture}

In this section we prove the first part of the Tate conjecture for $\mathbf{S}=S_{1} \times S_{2}$ :

Theorem 7.1. Let $k$ be a finite extension of $\mathbb{Q}$. Then we have

$$
\mathbf{U}\left(\pi_{1}, \pi_{2}, k\right)=\mathbf{V}\left(\pi_{1}, \pi_{2}, k\right),
$$

if either $\pi_{1}$ or $\pi_{2}$ is non-CM. 
We remark that by descent, it is sufficient to prove Theorem 7.1 for $k$ large. We assume that the representation $\pi_{1}$ is non-CM (the case when $\pi_{2}$ is non-CM is similar), and we prove Theorem 7.1 in this case.

We distinguish two cases:

A) The representation $\pi_{2}$ is CM. Thus $\rho_{\pi_{2}}=\operatorname{Ind}_{\Gamma_{M}}^{\Gamma_{F}} \chi$, where $M$ is a quadratic CM-extension of $F$ and $\chi$ is a character of $\Gamma_{M}$. From $\S 3$ we know that $\rho\left(\pi_{2}\right)$ is a subrepresentation of

that satisfies

$$
\operatorname{Ind}_{\Gamma_{F}}^{\Gamma_{\mathbb{Q}}}\left(\rho_{\pi_{2}} \otimes \rho_{\pi_{2}}^{\tau}\right)
$$

Thus, we deduce that

$$
\left.\rho\left(\pi_{2}\right)\right|_{\Gamma_{F}}=\rho_{\pi_{2}} \otimes \rho_{\pi_{2}}^{\tau}
$$

$$
\begin{gathered}
\left.\rho\left(\pi_{2}\right)\right|_{\Gamma_{k M M^{\tau}}} \cong(\chi \oplus \bar{\chi}) \otimes\left(\chi^{\tau} \oplus \bar{\chi}^{\tau}\right) \\
\cong \chi \chi^{\tau} \oplus \chi \bar{\chi}^{\tau} \oplus \bar{\chi} \chi^{\tau} \oplus \bar{\chi} \bar{\chi}^{\tau},
\end{gathered}
$$

where $\bar{\chi}$ is the complex conjugate of $\chi$.

From $\S 3$, we know that $\rho\left(\pi_{1}\right)$ is a subrepresentation of

$$
\operatorname{Ind}_{\Gamma_{F}}^{\Gamma_{\mathbb{Q}}}\left(\rho_{\pi_{1}} \otimes \rho_{\pi_{1}}^{\tau}\right)
$$

that satisfies

$$
\left.\rho\left(\pi_{1}\right)\right|_{\Gamma_{F}} \cong \rho_{\pi_{1}} \otimes \rho_{\pi_{1}}^{\tau}
$$

We distinguish two cases:

a) The representation $\left.\rho\left(\pi_{1}\right)\right|_{\Gamma_{F}}$ is irreducible. Then because $\left.\rho\left(\pi_{1}\right)\right|_{\Gamma_{F}} \cong \rho_{\pi_{1}} \otimes \rho_{\pi_{1}}^{\tau}$, and the representation $\pi_{1}$ is non-CM, from Propositions 5.1, 5.2, and 5.3, we deduce that the representation $\left.\rho\left(\pi_{1}\right)\right|_{\Gamma_{k M M} \tau}$ is irreducible and because $\left.\rho\left(\pi_{2}\right)\right|_{\Gamma_{k M M^{\tau}}}$ is a sum of 4 one-dimensional characters, we get that $\mathbf{V}\left(\pi_{1}, \pi_{2}, k\right)=\{0\}$, and thus $\mathbf{U}\left(\pi_{1}, \pi_{2}, k\right)=\mathbf{V}\left(\pi_{1}, \pi_{2}, k\right)=\{0\}$, and Theorem 7.1 is proved in this case.

b) The representation $\left.\rho\left(\pi_{1}\right)\right|_{\Gamma_{F}}$ is reducible. Then because $\left.\rho\left(\pi_{1}\right)\right|_{\Gamma_{F}} \cong \rho_{\pi_{1}} \otimes \rho_{\pi_{1}}^{\tau}$, and the representation $\pi_{1}$ is non-CM, applying Proposition 5.2, we get that $\rho_{\pi_{1}}^{\tau} \cong$ $\rho_{\pi_{1}} \otimes \alpha$ for some Hecke character $\alpha$ of $F$. Hence, from Proposition 5.4, we know that $\alpha$ is a Hecke character of $I_{F}$ which is trivial on $I_{\mathbb{Q}}$. Therefore $\alpha$ can be written as $\alpha=\chi^{\tau} / \chi$ for some Hecke character $\chi$ of $I_{F}$. Hence

$$
\left(\pi_{1} \otimes \chi^{-1}\right)^{\tau} \cong \pi_{1} \otimes \chi^{-1} .
$$

So $\pi_{1} \cong \pi_{0 / F} \otimes \chi$, where $\pi_{0 / F}$ is the base change to $F$ of some automorphic representation $\pi_{0}$ of $G L(2) / \mathbb{Q}$.

Then from the properties of $\rho\left(\pi_{1}\right)$ (see for example [MP]), we have

$$
\left.\rho\left(\pi_{1}\right) \cong\left(\operatorname{Sym}^{2} \rho_{\pi_{0}} \oplus \omega_{\pi_{0}} \cdot \omega_{F / \mathbb{Q}}\right) \otimes \chi\right|_{I_{\mathbb{Q}}},
$$

where $\omega_{\pi_{0}}$ is the central character of $\pi_{0}$ and $\omega_{F / \mathbb{Q}}$ is the quadratic character that corresponds to $F / \mathbb{Q}$.

Thus we get

$$
\begin{gathered}
\left.\rho\left(\pi_{1}\right)\right|_{\Gamma_{k N N^{\tau}}} \cong\left(\left.\left.\left.\operatorname{Sym}^{2} \rho_{\pi_{0}}\right|_{\Gamma_{k N N^{\tau}}} \otimes \chi\right|_{I_{\mathbb{Q}}}\right|_{\Gamma_{k N N^{\tau}}}\right) \\
\oplus\left(\left.\left.\left.\omega_{\pi_{0}}\right|_{\Gamma_{k N N^{\tau}}} \cdot \omega_{F k N N^{\tau} / k N N^{\tau}} \cdot \chi\right|_{I_{\mathbb{Q}}}\right|_{\Gamma_{k N N^{\tau}}}\right) .
\end{gathered}
$$

Since $\pi_{1}$ is non-CM and the representation $\pi_{0}$ is non-CM, from Proposition 5.1, we know that the representation $\left.\rho_{\pi_{0}}\right|_{\Gamma_{k N N} \tau}$ is irreducible and non-dihedral and from Proposition 5.5, we deduce that $\operatorname{Sym}^{2}\left(\left.\rho_{\pi_{0}}\right|_{\Gamma_{k N N \tau} \tau}\right)$ is irreducible. Because $\left.\rho\left(\pi_{2}\right)\right|_{\Gamma_{k N N^{\tau}}}$ is a sum of one-dimensional characters, we obtain that the dimension 
of $\mathbf{V}\left(\pi_{1}, \pi_{2}, k\right)$ is 0 or 1 , and it is equal to 1 precisely when the Tate cycles obtained are the product of Tate cycles of the individual factors $S_{1}$ and $S_{2}$ and thus algebraic, because from [HLR], $\mathrm{MR}$ ] and [K], we know that all the Tate cycles of $S_{1}$ and $S_{2}$ are algebraic. Hence in this case, Theorem 7.1 is proved.

B) The representation $\pi_{2}$ is non-CM. We distinguish two cases:

a) The representation $\left.\rho\left(\pi_{2}\right)\right|_{\Gamma_{F}}$ is irreducible. Then because $\left.\rho\left(\pi_{2}\right)\right|_{\Gamma_{F}} \cong \rho_{\pi_{2}} \otimes \rho_{\pi_{2}}^{\tau}$, and the representation $\pi_{2}$ is non-CM, from Propositions 5.1, 5.2, and 5.3, we deduce that the representation $\left.\rho\left(\pi_{2}\right)\right|_{\Gamma_{k F}}$ is irreducible. By Schur's lemma, we obtain that the dimension of $\mathbf{V}\left(\pi_{1}, \pi_{2}, k F\right)$ is 0 or 1 , and it is equal to 1 precisely when $\left.\left.\rho\left(\pi_{1}\right)\right|_{\Gamma_{k F}} \cong \rho\left(\pi_{2}^{*}\right)\right|_{\Gamma_{k F}}$. Hence $\left.\left.\left(\rho_{\pi_{1}} \otimes \rho_{\pi_{1}}^{\tau}\right)\right|_{\Gamma_{k F}} \cong\left(\rho_{\pi_{2}^{*}} \otimes \rho_{\pi_{2}^{*}}^{\tau}\right)\right|_{\Gamma_{k F}}$. Then from lemma 2.3 of [MP], and Proposition 5.1, we deduce that $\pi_{1} \otimes \mu \cong \pi_{2}^{*}$, for some character $\mu$ of $\Gamma_{F}$. Then from the properties of the representations $\rho\left(\pi_{1}\right)$ and $\rho\left(\pi_{2}\right)$, we get that the space $V\left(\pi_{1}\right) \otimes V\left(\pi_{2}\right)$ has only one subspace of dimension 1 , namely $\left.\mu^{-1}\right|_{I_{\mathbb{Q}}}$. Let $\left.\mathbb{Q}^{\mu}\right|_{I_{\mathbb{Q}}}$ be the abelian extension of $\mathbb{Q}$ defined by $\left.\mu\right|_{I_{\mathbb{Q}}}$. Then all the Tate cycles of $V\left(\pi_{1}\right) \otimes V\left(\pi_{2}\right)$ are defined over $\mathbb{Q}^{\left.\mu\right|_{I_{\mathbb{Q}}}}$.

We have that $\pi_{1} \otimes \mu \cong \pi_{2}^{*}$, and because for any $\pi$ as above, $\rho(\pi)$ is automorphic (see $[\mathrm{R}]$ ), from Proposition 5.8, we deduce that $L\left(s, \rho\left(\pi_{1} \otimes \mu\right) \otimes \rho\left(\pi_{2}\right)\right)$ has a pole at $s=1$, which implies by the residue formula that

$$
\int_{G L(2, F) Z\left(\mathbb{A}_{F}\right) \backslash G L\left(2, \mathbb{A}_{F}\right)} \phi_{1}(g) \phi_{2}(g) \mu(\operatorname{det}(g)) d g \neq 0,
$$

for some function $\phi_{1}$ in the space of $\pi_{1}$ and some function $\phi_{2}$ in the space of $\pi_{2}$, where $Z$ denotes the center of $G L(2)$. In other words, the integral of the $(2,2)$-form $\eta_{\phi_{1}, \phi_{2}}=\left((2 \pi i)^{2} \phi_{1}\left(z_{1}, z_{2}\right) d z_{1} \wedge \overline{d z_{2}}\right) \wedge\left((2 \pi i)^{2} \phi_{2}\left(z_{3}, z_{4}\right) d z_{3} \wedge \overline{d z_{4}}\right)$ on $S_{K_{1}} \times S_{K_{2}}$ defined by $\left(\phi_{1}, \phi_{2}\right)$ has a non-zero twisted period over $S_{K_{1}}$. Then the corresponding twisting correspondence (see $\S 4$ ) of $S_{K_{1}} \times S_{K_{2}}$ defines (for suitable $g_{1}$ and $g_{2}$ in $G\left(\mathbb{A}_{f}\right)$ ) a $\mu$-twisted cycle $Z(\mu)=Z_{g_{1}, g_{2}}(\mu)$ of codimension 2 of $S_{K_{1}} \times S_{K_{2}}$, and we get that

$$
\int_{Z(\mu)} \eta_{\phi_{1}, \phi_{2}} \neq 0
$$

and thus $Z(\mu)$ is homologically non-trivial and we get

$$
\mathbf{U}\left(\pi_{1}, \pi_{2} ;\left.\mu\right|_{I_{\mathbb{Q}}}\right) \neq\{0\} .
$$

Hence both spaces $\mathbf{U}\left(\pi_{1}, \pi_{2} ;\left.\mu\right|_{I_{\mathbb{Q}}}\right)$ and $\mathbf{V}\left(\pi_{1}, \pi_{2} ;\left.\mu\right|_{I_{\mathbb{Q}}}\right)$ have dimension 1 and are equal. Thus, if $k$ contains $\mathbb{Q}^{\left.\mu\right|_{\mathbb{Q}}}$, both spaces $\mathbf{U}\left(\pi_{1}, \pi_{2}, k\right)$ and $\mathbf{V}\left(\pi_{1}, \pi_{2}, k\right)$ have dimension 1 and are equal, and Theorem 7.1 is proved in this case.

b) The representation $\left.\rho\left(\pi_{2}\right)\right|_{\Gamma_{F}}$ is reducible. If $\mathbf{V}\left(\pi_{1}, \pi_{2}, k F\right)=\{0\}$, we get that $\mathbf{U}\left(\pi_{1}, \pi_{2}, k\right)=\mathbf{V}\left(\pi_{1}, \pi_{2}, k\right)=\{0\}$, and Theorem 7.1 is proved in this case. We assume from now on that $\mathbf{V}\left(\pi_{1}, \pi_{2}, k F\right) \neq\{0\}$, which implies that $\left.\rho\left(\pi_{1}\right)\right|_{\Gamma_{F k}}$ is reducible. Since $\pi_{2}$ is non-CM, from Propositions 5.1 and 5.2, we get that $\rho_{\pi_{2}}^{\tau} \cong$ $\rho_{\pi_{2}} \otimes \beta$ for some Hecke character $\beta$ of $F$. Hence, from Proposition 5.4, we know that $\beta$ is a Hecke character of $I_{F}$ which is trivial on $I_{\mathbb{Q}}$. Therefore $\beta$ can be written as $\beta=\psi^{\tau} / \psi$ for some Hecke character $\psi$ of $I_{F}$. Hence

$$
\left(\pi_{2} \otimes \psi^{-1}\right)^{\tau} \cong \pi_{2} \otimes \psi^{-1} .
$$

So $\pi_{2} \cong \pi_{0 / F}^{\prime} \otimes \psi$, where $\pi_{0 / F}^{\prime}$ is the base change to $F$ of some automorphic representation $\pi_{0}^{\prime}$ of $G L(2) / \mathbb{Q}$. 
Then from the properties of $\rho\left(\pi_{2}\right)$ (see for example [MP]), we have

$$
\left.\rho\left(\pi_{2}\right) \cong\left(\operatorname{Sym}^{2} \rho_{\pi_{0}^{\prime}} \oplus \omega_{\pi_{0}^{\prime}} \cdot \omega_{F / \mathbb{Q}}\right) \otimes \psi\right|_{I_{\mathbb{Q}}},
$$

where $\omega_{\pi_{0}^{\prime}}$ is the central character of $\pi_{0}^{\prime}$, and $\omega_{F / \mathbb{Q}}$ is the quadratic character that corresponds to $F / \mathbb{Q}$.

Also since $\left.\rho\left(\pi_{2}\right)\right|_{\Gamma_{F}}$ is reducible, with the same notation as in case $\left.1, \mathrm{~b}\right)$, we get that $\pi_{1} \cong \pi_{0 / F} \otimes \mu$ and that

$$
\left.\rho\left(\pi_{1}\right) \cong\left(\operatorname{Sym}^{2} \rho_{\pi_{0}} \oplus \omega_{\pi_{0}} \cdot \omega_{F / \mathbb{Q}}\right) \otimes \mu\right|_{I_{\mathbb{Q}}} .
$$

Now, since $\pi_{1}$ and $\pi_{2}$ are non-CM, also $\pi_{0}$ and $\pi_{0}^{\prime}$ are non-CM, and thus, from Proposition 5.5, we get that the representations $\operatorname{Sym}^{2} \rho_{\pi_{0}}$ and $\operatorname{Sym}^{2} \rho_{\pi_{0}^{\prime}}$ are irreducible. Hence for all $k$ sufficiently large, $\mathbf{V}\left(\pi_{1}, \pi_{2}, k\right)$ has dimension 1 or 2 , and it has dimension 2 if and only if $\left.\left.\operatorname{Sym}^{2} \rho_{\pi_{0}^{\prime *}}\right|_{\Gamma_{k}} \cong \operatorname{Sym}^{2} \rho_{\pi_{0}}\right|_{\Gamma_{k}} \otimes \eta$ for some character $\eta$ of $\Gamma_{k}$. If for $k$ sufficiently large $\mathbf{V}\left(\pi_{1}, \pi_{2}, k\right)$ has dimension 1 , then the Tate cycles are obtained as a tensor product of Tate cycles of the individual factors $S_{1}$ and $S_{2}$ and thus are algebraic, because from [HLR, [MR and [K], we know that all the Tate cycles of $S_{1}$ and $S_{2}$ are algebraic, and thus Theorem 7.1 is proved in this case. We assume from now on that for $k$ sufficiently large, $\mathbf{V}\left(\pi_{1}, \pi_{2}, k\right)$ has dimension 2. Then from Propositions 5.1, 5.7 and 5.3, we deduce that $\pi_{0} \otimes \gamma \cong \pi_{0}^{\prime *}$ for some character $\gamma$ of $\Gamma_{\mathbb{Q}}$. Hence $\left.\pi_{1} \otimes \gamma\right|_{\Gamma_{F}} \cdot(\mu \psi)^{-1} \cong \pi_{2}^{*}$. Then $\rho\left(\pi_{1}\right) \otimes \rho\left(\pi_{2}\right)$ contains in its decomposition as a direct sum of irreducible representations, only two one-dimensional representations, namely $\left.\gamma^{-1} \cdot(\mu \psi)\right|_{I_{\mathbb{Q}}}$ and $\left.\gamma^{-1} \cdot(\mu \psi)\right|_{I_{\mathbb{Q}}}$. Let $\mathbb{Q}^{\left.\gamma^{-1} \cdot(\mu \psi)\right|_{I_{\mathbb{Q}}}}$ be the abelian extension of $\mathbb{Q}$ defined by $\left.\gamma^{-1} \cdot(\mu \psi)\right|_{I_{\mathbb{Q}}}$. Then all the Tate cycles of $V\left(\pi_{1}\right) \otimes V\left(\pi_{2}\right)$ are defined over $\mathbb{Q}^{\left.\gamma^{-1} \cdot(\mu \psi)\right|_{\mathbb{Q}}}$. Only one of these two Tate cycles corresponding to $\left.\gamma^{-1} \cdot(\mu \psi)\right|_{I_{\mathbb{Q}}}$ is obtained as a tensor product of Tate cycles of the individual factors $S_{1}$ and $S_{2}$ and thus is algebraic, because from [HLR, [MR] and [K], we know that all the Tate cycles of $S_{1}$ and $S_{2}$ are algebraic. Hence we know that $\mathbf{V}\left(\pi_{1}, \pi_{2} ;\left.\gamma \cdot(\mu \psi)^{-1}\right|_{I_{\mathbb{Q}}}\right)$ has dimension 2 and $\mathbf{U}\left(\pi_{1}, \pi_{2} ;\left.\gamma \cdot(\mu \psi)^{-1}\right|_{I_{\mathbb{Q}}}\right)$ has dimension at least 1 .

But $\left.\pi_{1} \otimes \gamma\right|_{\Gamma_{F}} \cdot(\mu \psi)^{-1} \cong \pi_{2}^{*}$, and because of the above decompositions of $\rho\left(\pi_{1}\right)$ and $\rho\left(\pi_{2}\right)$, from Propositions 5.6 and 5.8, we deduce that $L\left(s, \rho\left(\left.\pi_{1} \otimes \gamma\right|_{\Gamma_{F}} \cdot(\mu \psi)^{-1}\right) \otimes\right.$ $\left.\rho\left(\pi_{2}\right)\right)$ has a pole of order 2 at $s=1$, which implies as above in the case a), that $Z\left(\left.\gamma\right|_{\Gamma_{F}} \cdot(\mu \psi)^{-1}\right)$ is homologically non-trivial and thus is a non-zero algebraic cycle of $\mathbf{U}\left(\pi_{1}, \pi_{2} ;\left.\gamma^{-1} \cdot(\mu \psi)\right|_{I_{Q}}\right)$.

Lemma 7.2. There exists a finite order character $\xi$ of $F$ such that

(i) $s(\xi)=(-,-)$,

(ii) $\left.\xi\right|_{I_{\mathbb{Q}}}=1$.

Proof. Let $\lambda$ be a finite order character of $F$ of signature $(+,-)$. Then $\lambda^{\tau}$ has signature $(-,+)$. Set $\xi=\lambda / \lambda^{\tau}$. Then we have that $s(\xi)=(-,-)$ and $\left.\xi\right|_{I_{\mathbb{Q}}}=1$, because $\mathbb{Q}$ is the fixed field of $\tau$.

From the properties of $\rho(\pi)$ for $\pi$ as above, we know that for any character $\eta$ of $\Gamma_{F}$, we have $\rho(\pi \otimes \eta)=\left.\rho(\pi) \otimes \eta\right|_{I_{\mathbb{Q}}}$. Hence if we choose $\xi$ as in Lemma 7.2 , since $\left.\xi\right|_{I_{Q}}=1$, we get that $\rho(\pi \otimes \xi)=\rho(\pi)$ for any $\pi$. Thus

$$
L\left(s, \rho\left(\left.\pi_{1} \otimes \xi \gamma\right|_{\Gamma_{F}} \cdot(\mu \psi)^{-1}\right) \otimes \rho\left(\pi_{2}\right)\right)=L\left(s, \rho\left(\left.\pi_{1} \otimes \gamma\right|_{\Gamma_{F}} \cdot(\mu \psi)^{-1}\right) \otimes \rho\left(\pi_{2}\right)\right)
$$


has a pole of order 2 at $s=1$, which implies as above that $Z\left(\left.\xi \gamma\right|_{\Gamma_{F}} \cdot(\mu \psi)^{-1}\right)$ is homologically non-trivial and thus is a non-zero algebraic cycle in $\mathbf{U}\left(\pi_{1}, \pi_{2} ; \gamma^{-1}\right.$. $\left.\left.(\mu \psi)\right|_{I_{Q}}\right)$. Hence in $\mathbf{U}\left(\pi_{1}, \pi_{2} ;\left.\gamma^{-1} \cdot(\mu \psi)\right|_{I_{Q}}\right)$ we have two homologically non-trivial algebraic cycles $Z\left(\left.\xi \gamma\right|_{\Gamma_{F}} \cdot(\mu \psi)^{-1}\right)$ and $Z\left(\left.\gamma\right|_{\Gamma_{F}} \cdot(\mu \psi)^{-1}\right)$. But these two algebraic cycles are not proportional, because from Lemma 7.2, we know that $s(\xi)=(-,-)$, and thus $s\left(\left.\xi \gamma\right|_{\Gamma_{F}} \cdot(\mu \psi)^{-1}\right) \neq s\left(\left.\gamma\right|_{\Gamma_{F}} \cdot(\mu \psi)^{-1}\right)$, and from Lemma 4.1, we know that the twisting correspondence $R\left(\left.\xi \gamma\right|_{\Gamma_{F}} \cdot(\mu \psi)^{-1}\right)$ sends a vector in $V_{B}\left(\pi_{1}\right)^{s}$ into $V_{B}\left(\left.\pi_{1} \otimes \xi \gamma\right|_{\Gamma_{F}} \cdot(\mu \psi)^{-1}\right)^{s s\left(\left.\xi \gamma\right|_{\Gamma_{F}} \cdot(\mu \psi)^{-1}\right)}=V_{B}\left(\left.\pi_{1} \otimes \gamma\right|_{\Gamma_{F}} \cdot(\mu \psi)^{-1}\right)^{s s\left(\left.\xi \gamma\right|_{\Gamma_{F}} \cdot(\mu \psi)^{-1}\right)}$, and the twisting correspondence $R\left(\left.\gamma\right|_{\Gamma_{F}} \cdot(\mu \psi)^{-1}\right)$ sends a vector in $V_{B}\left(\pi_{1}\right)^{s}$ into $V_{B}\left(\left.\pi_{1} \otimes \gamma\right|_{\Gamma_{F}} \cdot(\mu \psi)^{-1}\right)^{s s\left(\left.\gamma\right|_{\Gamma_{F}} \cdot(\mu \psi)^{-1}\right)}$. Hence $\mathbf{U}\left(\pi_{1}, \pi_{2} ;\left.\gamma^{-1} \cdot(\mu \psi)\right|_{I_{Q}}\right)$ has dimension 2, and we obtain that both spaces $\mathbf{U}\left(\pi_{1}, \pi_{2} ;\left.\gamma^{-1} \cdot(\mu \psi)\right|_{I_{\mathbb{Q}}}\right)$ and $\mathbf{V}\left(\pi_{1}, \pi_{2} ;\left.\gamma^{-1} \cdot(\mu \psi)\right|_{I_{\mathbb{Q}}}\right)$

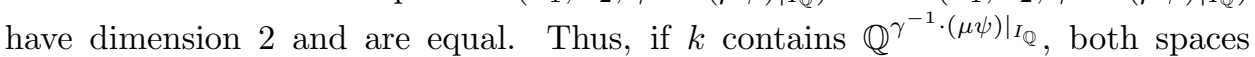
$\mathbf{U}\left(\pi_{1}, \pi_{2}, k\right)$ and $\mathbf{V}\left(\pi_{1}, \pi_{2}, k\right)$ have dimension 2 and are equal, and Theorem 7.1 is proved in this case.

\section{Poles of $L$-FunCtions}

Proposition 8.1. If $k$ is a solvable extension of $\mathbb{Q}$, then the order of the pole at $s=1$ of $L\left(s,\left.\left.\rho\left(\pi_{1}\right)\right|_{\Gamma_{k}} \otimes \rho\left(\pi_{2}\right)\right|_{\Gamma_{k}}\right)$ is equal to $\operatorname{dim}_{\overline{\mathbb{Q}}_{l}} \mathbf{V}\left(\pi_{1}, \pi_{2}, k\right)$.

Proof. From $\left[\mathbf{R}\right.$, we know that the representations $\rho\left(\pi_{1}\right)$ and $\rho\left(\pi_{2}\right)$ are automorphic, and thus using the Langlands base change $[\mathrm{L}$ and the decompositions of the representations $\rho\left(\pi_{1}\right)$ and $\rho\left(\pi_{2}\right)$ from $\S 7$, and using Propositions 5.6, 5.8 and 5.9, one obtains easily Proposition 8.1.

\section{REFERENCES}

[D] P. Deligne, Travaux de Shimura, Sém. Bourbaki Féb. 71, Exposé 389, Lectures Notes in Math. vol. 244. Berlin-Heidelberg-New York; Springer, 1971. MR0498581 (58:16675)

[FH] Y. Z. Flicker, J. L. Hakim, Quaternionic Distinguished Representations, American J. of Math., Vol. 116, No. 3. (Jun., 1994), 683-736. MR.1277452 (95i:22028)

[HLR] G. Harder, R. P. Langlands, M. Rapoport, Algebraische Zycklen auf Hilbert-BlumenthalFlächen, J. Reine Angew. Math. 366, 53-120 (1986). MR833013 (87k:11066)

[G] G. van der Geer, Hilbert modular surfaces, Springer-Verlag, 1988. MR.930101 (89c:11073)

[GJ] S. Gelbart, H. Jacquet, A relation between automorphic representations of $G L(2)$ and GL(3), Ann. Sci. École Norm. Sup. 11(1979),471-542. MR533066 (81e:10025)

[JPSS] H. Jacquet, I. I. Piatetski-Shapiro, J. A. Shalika, Rankin-Selberg convolutions, American J. of Math., 105, nr. 2 (1983), 367-464. MR701565 (85g:11044)

[K] C. Klingenberg, Die Tate-Vermutungen für Hilbert-Blumenthal-Flächen, Invent. Math. 89, 291-317(1987). MR894381 (88m:11042)

[L] R. P. Langlands, Base change for GL(2), Ann. of Mathematics Studies 96, Princeton University Press, 1980. MR574808 (82a:10032)

[LA] K. F. Lai, Algebraic cycles on compact Shimura surface, Math. Z. 189, 593-602 (1985). MR:786286 (87a:11057)

[MP] V. K. Murty, D. Prasad, Tate cycles on a product of two Hilbert modular surfaces, J. Number Theory 80(1)(2000) 25-43. MR.1735646 (2000m:14028)

[MR] V. K. Murty, D. Ramakrishnan, Period relations and the Tate conjecture for Hilbert modular surfaces, Invent. Math. 89, 319-345(1987). MR894382 (89a:11064)

[R] D. Ramakrishnan, Modularity of solvable Artin representations of GO(4)-type, IMRN 2002, No. 1, 1-54. MR1874921(2003b:11049)

[RT] J. D. Rogawski, J. B. Tunnell, On Artin L-functions associated to Hilbert modular forms of weight one, Invent. Math. 74, 1983, 1-43. MR722724 (85i:11044) 
[T] R. Taylor, On Galois representations associated to Hilbert modular forms, Invent. Math. 98, 1989, 265-280. MR 1016264 (90m:11176)

[TA] J. Tate, Algebraic cycles and poles of zeta functions, In: Schilling, O.D.G. (ed.), Arithmetical algebraic geometry, New York: Harper and Row, 1966. MR.0225778 (37:1371)

Department of Mathematics, Columbia University, New York, New York 10027 